\title{
Individual differences in affective flexibility predict future anxiety and worry
}

\section{Eve Twivy , Maud Grol \& Elaine Fox}

To cite this article: Eve Twivy , Maud Grol \& Elaine Fox (2020): Individual differences in affective flexibility predict future anxiety and worry, Cognition and Emotion, DOI: 10.1080/02699931.2020.1843407

To link to this article: https://doi.org/10.1080/02699931.2020.1843407

曲 Published online: 05 Nov 2020.

Submit your article to this journal $\pi$

山 Article views: 138

Q View related articles $\widetilde{ }$

View Crossmark data $־$ 


\title{
Individual differences in affective flexibility predict future anxiety and worry
}

\author{
Eve Twivy*, Maud Grol*† and Elaine Fox \\ Department of Experimental Psychology, University of Oxford, Oxford, United Kingdom
}

\begin{abstract}
Deficits in cognitive flexibility have been associated with anxiety and worry, however few studies have assessed cognitive flexibility in the context of emotional stimuli (i.e. affective flexibility). The present study $(n=79)$ investigated whether individual differences in affective flexibility predict levels of trait anxiety and worry over a period of seven weeks. Affective flexibility was measured using a task-switching paradigm. Results showed that less efficient shifting of attention towards affective aspects of positive stimuli predicted higher anxiety over time. Additionally, more efficient shifting of attention away from affective towards non-affective aspects of negative stimuli predicted higher anxiety and worry over time. This latter finding may be understood by considering theoretical models and empirical evidence associating avoidance of negative information with increased anxiety. The effects were small and require replication in larger, representative samples, but they are an initial indication that anxiety may not be associated with general impairments in cognitive flexibility. Instead, our study emphasises the importance of breaking down cognitive flexibility into different components to investigate more nuanced relationships.
\end{abstract}

ARTICLE HISTORY

Received 14 May 2020

Revised 15 October 2020

Accepted 24 October 2020

\section{KEYWORDS}

Cognitive flexibility; affective flexibility; anxiety; worry
Cognitive flexibility can be defined as the ability to shift attention according to changing situational demands (Geurts et al., 2009) and is considered crucial for adaptive behaviour because it enables one to flexibly respond to the ever-changing environment (Kashdan \& Rottenberg, 2010). For example, focusing on potential threats may be adaptive in some situations, such as crossing the road, but maladaptive in other situations, such as chatting to friends. Thus, the ability to shift attention efficiently enables and supports context-appropriate behaviour.

Cognitive flexibility is typically operationalised as the ability to switch between task rules and is generally thought to rely on inhibition of the irrelevant task and shifting to the new task (Monsell, 2003). Deficits in cognitive flexibility have been associated with generalised anxiety disorder (Lee \& Orsillo, 2014) as well as high trait anxiety in non-clinical populations (Ansari et al., 2008; Mărcuş et al., 2016). However, there have been some failures to replicate this association (Han et al., 2016). A potential problem with this research is that cognitive flexibility has typically been assessed with tasks using non-emotional stimuli. Given that anxiety and worry are associated with aberrant processing of affective information, it is particularly relevant to explore the relationship with cognitive flexibility in the context of emotionally charged information. More specifically, assessing the ability to shift between processing the affective and non-affective aspects of emotional material according to changing situational demands (i.e. affective flexibility: Malooly et al., 2013) is important.

CONTACT Maud Grol maudgrol@gmail.com @D Department of Experimental Clinical and Health Psychology, Ghent University, Henri Dunantlaan 2, 9000 Ghent, Belgium

*Equal contributions

${ }^{\dagger}$ Present address Maud Grol: Department of Experimental Clinical and Health Psychology, Ghent University, Belgium. 
Cognitive models propose that anxiety and worry are associated with selective biases in information processing and poorer top-down cognitive control (Eysenck et al., 2007; Hirsch \& Mathews, 2012). For example, trait anxiety is typically associated with initial facilitation of attention to negative or threatrelated information, which is then followed by increased avoidance of such information, particularly at longer exposure intervals (Mogg et al., 2004; for review see Cisler \& Koster, 2010). This attentional avoidance possibly reflects a strategic process underlying emotion regulation to reduce arousal (Cisler \& Koster, 2010). Whilst substantial research has investigated information processing biases related to trait anxiety, other research has focused on whether anxiety and worry are associated with deficits in cognitive control, i.e. the set of cognitive processes that enable goal-directed behaviour. Attentional control theory (ACT) predicts that anxiety will impair efficiency on cognitive tasks that rely on inhibition or shifting processes of working memory (Eysenck et al., 2007), especially in the presence of salient emotional stimuli (for a review, see Berggren \& Derakshan, 2013). Given that cognitive flexibility relies on both inhibition and shifting, ACT indirectly predicts that high trait anxiety will be associated with poorer cognitive flexibility, particularly affective flexibility.

Few studies have investigated the relationship between affective flexibility and anxiety. As an example, one study found that poorer affective flexibility was associated with higher trait anxiety in adolescents, and proposed that inflexibility may act a risk factor in the development of high trait anxiety (Mărcuş et al., 2016). However, cross-sectional studies are unable to shed light on whether differences in affective flexibility are linked to changes in anxiety and worry. Moreover, most studies have measured cognitive or affective flexibility as a single construct, without considering the emotional context and whether one is shifting towards or away from affective aspects of emotional material.

Research which has broken down affective flexibility into different components has started to reveal more nuanced relationships. For example, reduced flexibility when shifting away from the affective and towards the non-affective aspects of negative information was associated with decreased reappraisal effectiveness (Malooly et al., 2013) and increased rumination (Genet et al., 2013). Less efficient shifting towards the affective aspects of positive material has also been associated with decreased reappraisal effectiveness (Malooly et al., 2013), while more efficient shifting towards the non-affective aspects of positive stimuli has been associated with increased rumination. This latter finding suggest that more efficient set-shifting may not be adaptive per se (Genet et al., 2013).

\section{The present study}

The present study was designed to investigate whether individual differences in affective flexibility would predict higher levels of trait anxiety and worry over a seven-week period. We utilised a nonclinical population and conceptualised anxiety and worry as continuous dimensions. Whilst worry is a core cognitive feature of anxiety, we measured both to examine whether affective flexibility was also related to worry (as a transdiagnostic process) in isolation. Previous work has provided compelling evidence that trait measures can change over time in response to both clinical and nonclinical interventions (for a review see Roberts et al., 2017), and that changes across a period of weeks can be related to attentional biases (e.g. Clarke et al., 2008). Specifically, the readiness to acquire a bias in attention towards threatening stimuli has been shown to predict increases in trait anxiety in students, in response to extended stress during the university semester (Clarke et al., 2008). A recent meta-analysis that tracked changes across the Big Five trait categories in response to clinical and nonclinical interventions, has also shown that especially neuroticism, or emotional stability reversed, showed the largest changes, also across shorter periods of time (Roberts et al., 2017).

Affective flexibility was measured at baseline using a previously developed task-switching task (Genet et al., 2013; Malooly et al., 2013), in which participants were required to switch between categorising emotional images according to affective (valence) or non-affective aspects (number of people depicted).

Previous findings have related poorer cognitive flexibility to higher trait anxiety (Ansari et al., 2008; Mărcuş et al., 2016), and specifically reduced flexibility when shifting away from the affective aspects of negative information to increased rumination in daily life (Genet et al., 2013). Therefore, we hypothesised that reduced flexibility when shifting away from processing affective aspects towards nonaffective aspects of negative stimuli would predict higher anxiety and worry over a seven-week period. 
Emerging research also relates reduced attentional bias towards positive stimuli with greater anxiety vulnerability (Taylor et al., 2011). Therefore, we also hypothesised that reduced flexibility when shifting attention from processing non-affective aspects towards affective aspects of positive stimuli would predict higher anxiety and worry over time.

\section{Method}

\section{Participants}

Eighty participants were recruited by convenience sampling, through online message boards from the university. ${ }^{1}$ There was one drop out during the follow-up stages, leaving 79 participants (49 women, 1 of unspecified gender) aged between 18 and 40 years old $(M=22.37$ years, $S D=4.40)$, with $86.1 \%$ having (or studying for) an undergraduate degree or higher. Participants received monetary compensation or course credits for their participation. The study received ethical approval from the local Medical Sciences Interdivisional Research Ethics Committee (Reference: R49476/RE001).

\section{Materials}

\section{Self-report questionnaires ${ }^{2}$}

Trait anxiety was measured using the trait component of the State-Trait Anxiety Inventory (STAI-T; Spielberger et al., 1983) The STAI-T consists of 20 statements (e.g. "I feel nervous and restless") that participants rate according to how they generally feel on a 4-point scale from 1 (almost never) to 4 (almost always). A total score is obtained between 20 and 80 . In the present study, the baseline STAI-T measure $(\alpha=.94)$ and final STAI-T measure $(a=.94)$ had high internal consistency. The STAI-T had good test-retest reliability $(r=.86)$. Although the STAI-T is usually described as a measure of trait anxiety, previous results also suggest that the scale does not strictly assess anxiety, but rather negative affect in general (Bados et al., 2010).

Trait worry was measured using the Penn State Worry Questionnaire (PSWQ; Meyer et al., 1990), which consists of 16 statements (e.g. "I worry all the time") that participants rated on a 5-point scale from 1 (not at all typical of me) to 5 (very typical of me). A total score is obtained between 16 and 80 . In the present study, the baseline PSWQ measure $(a=.94)$ and final PSWQ measure $(a=.94)$ had high internal consistency. The PSWQ had good test-retest reliability $(r=.86)$.

\section{Affective flexibility task}

A task-switching task developed by Malooly et al. (2013) was used to measure affective flexibility. Participants were required to categorise a series of emotional images according to an affective rule (positive or negative) or a non-affective rule $(\leq 1$ or $\geq 2$ people depicted). Images were selected from the International Affective Picture System (IAPS; Lang et al., 1999). Forty images were used for each of the following four categories: negative with two or more people, negative with one or fewer people, positive with two or more people and positive with one or fewer people (160 images in total). Negative images consisted of both threat and non-threat (sadness/ loss-based) images. Mean valence for positive (range $=6.40-8.34, M=7.41, S D=.41$ ) and negative images $\quad($ range $=1.67-3.81, \quad M=2.78, \quad S D=.55$ ) differed significantly, $t(146.27)=60.3, p<.001, d=$ 9.53, whilst the mean arousal ratings did not differ for positive $(M=5.13, S D=.71)$ and negative images $(M=5.33, S D=.80), t(158)=1.63, p=.104, d=0.26$. An additional 20 images were used for the practice blocks.

In each trial a black screen appeared for $250 \mathrm{~ms}$ followed by a fixation cross in the centre of the screen, also for $250 \mathrm{~ms}$ (see Figure 1). Next, an image was presented centrally until the participant responded or for $5000 \mathrm{~ms}$, whichever was sooner. Cues indicating the relevant task appeared on either side of the image: " + " and " - " for the affective rule and " $\leq 1$ " or " $\geq 2$ " for the non-affective rule. The colour of the frame around the image (grey or white) also indicated sorting rule. The categorisation was performed by pressing one of two adjacent keyboard keys labelled as $L$ and $R$, corresponding to the cue to the left and right of the image.

Participants were instructed to work quickly but try to be as accurate as possible. There were eight task versions, counterbalancing different combinations of cue to response key mappings and task rule to background colour mappings. The task started with two 10-trial practice blocks, first only the affective categorisation and then only non-affective categorisation. There were two 160-trial test blocks, in which trials were presented in a pseudorandom order (Malooly et al., 2013). 


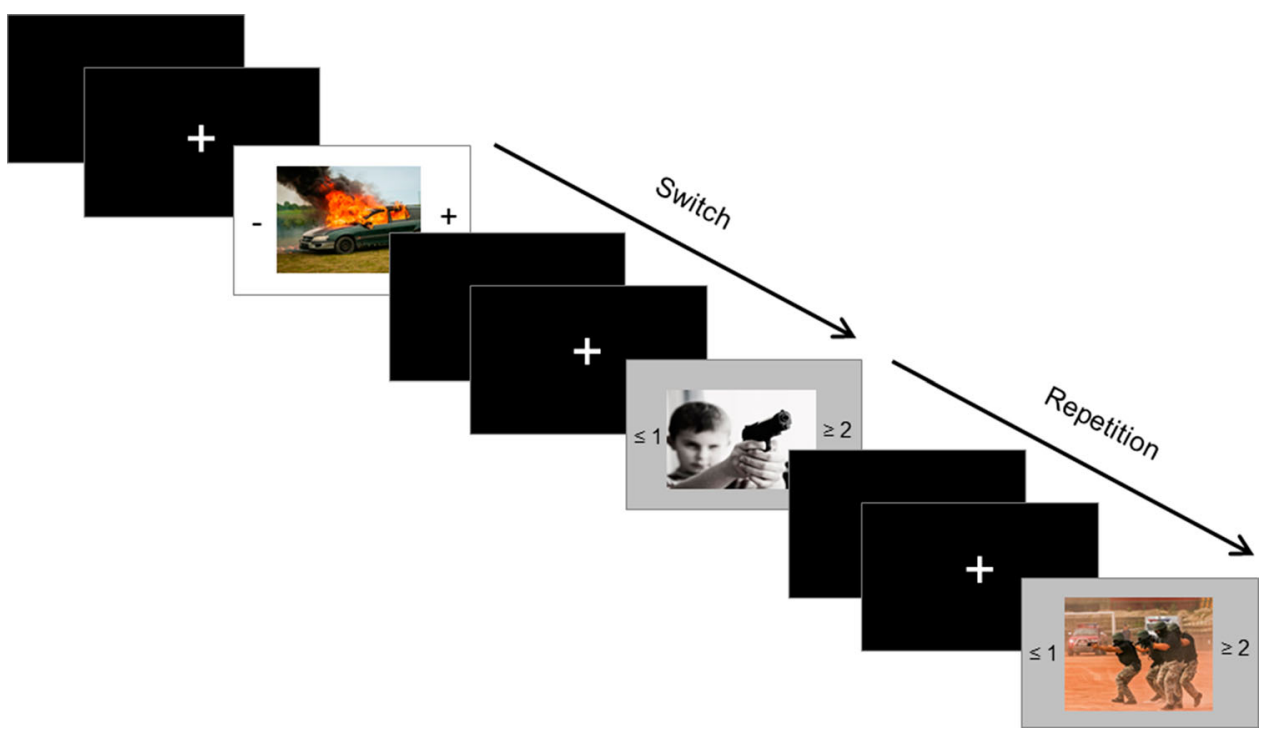

Figure 1. An example of a trial sequence. The switch trial is a negative non-affective switch. The images displayed are from the Open Affective Standardised Image Set (www.benedekkurdi.com/\#oasis).

Switch costs were calculated by subtracting the average reaction time (RT) on repetition trials from the average RT on switch trials, where higher switch costs reflect less efficient shifting (i.e. poorer flexibility). Following the method outlined by Malooly et al. (2013), four different types of switch costs
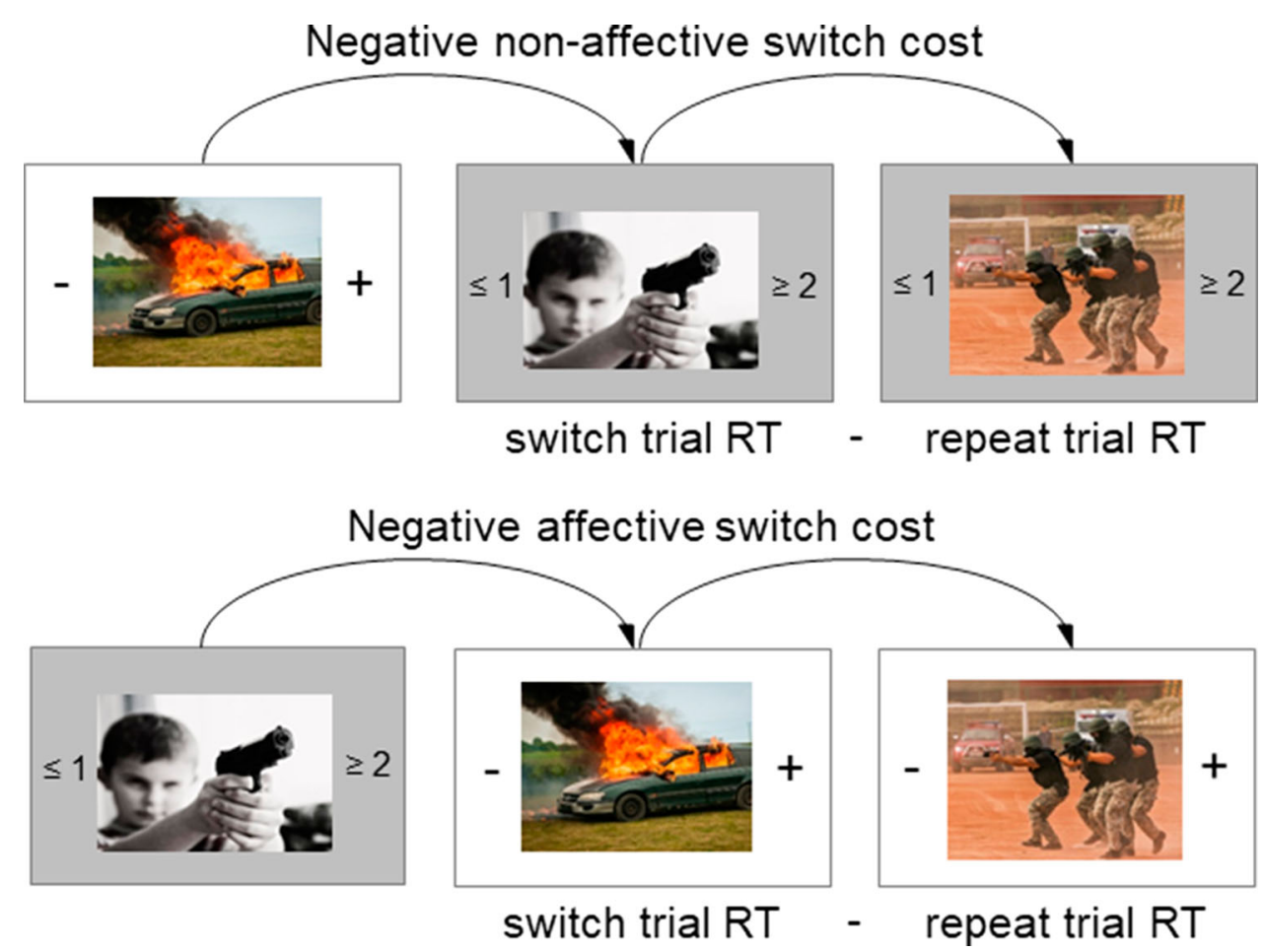

Figure 2. Calculation of specific switch costs, negative switch types are illustrated. The images displayed are from the Open Affective Standardized Image Set (www.benedekkurdi.com/\#oasis). 
were calculated. For example, negative non-affective switch costs were calculated by subtracting the average RT on trials involving a repetition of the non-affective categorisation in the context of negative images, from the average RT on trials involving a switch from categorising the affective aspect to categorising the non-affective aspect in the context of negative images (see Figure 2). Negative affective switch costs were then calculated by subtracting the average RT on trials involving a repetition of the affective categorisation in the context of negative images, from the average RT on trials involving a switch from categorising the non-affective aspect to categorising the affective aspect in the context of negative images. Positive non-affective and affective switch costs were calculated the same way, using trials in the context of positive images.

We calculated internal consistency, employing a permutation based split-half reliability approach with the R package "splithalf", using 5000 random splits (Parsons, 2020). Spearman-Brown corrected reliability estimates for $\mathrm{RT}$ within each of the trial types used to calculate the four switch costs, showed acceptable internal consistency ranging between 0.73 and 0.82 .

\section{Procedure}

During the first session participants completed the self-report questionnaires, followed by the affective flexibility task. Seven weeks after the initial session, participants again completed the self-report questionnaires. Following the final session, participants were debriefed by email.

\section{Data analysis}

To test whether affective flexibility predicts levels of STAI-T trait anxiety and PSWQ worry over a sevenweek period, we conducted hierarchical multiple regression analyses on the final STAI-T/PSWQ score. We first entered baseline STAI-T/PSWQ score, in a second step we entered negative non-affective switch costs and positive affective switch costs. Although our hypotheses focussed on these switch types, in a third step we entered the other switch costs, to explore the relationship of negative affective switch costs and positive non-affective switch costs with STAI-T trait anxiety/ PSWQ worry.

Previous research using this task (Genet et al., 2013; Malooly et al., 2013) has focussed on RT based switch costs reflecting the efficiency of shifting between affective and non-affective aspects of emotional material. Moreover, Attentional Control Theory has proposed that anxiety is associated with impaired efficiency to shift between tasks rather than impaired ability (Berggren \& Derakshan, 2013). Therefore, our main analyses focus on RT-based switch costs.

\section{Results}

\section{Preliminary analysis}

In the affective flexibility task, we only included trials that were preceded by a trial with a correct response (Demanet et al., 2011), because if the preceding trial is incorrect it is unclear whether the current trial is a repetition or switch from the participant's perspective. Therefore, we calculated accuracy by dividing the total number of correct responses that were preceded by a correct response, by the total number of (correct and incorrect) trials that were preceded by a correct response.

Only correct trials, preceded by a correct response, were used to calculate the mean $\mathrm{RT}$, resulting in an average deletion of $13.8 \%$ of trials for each participant. To reduce the influence of outliers, RT values 2.5 SD above and below the individual's mean RT on a specific trial type were replaced by the upper and lower cut-off values, following previous studies (e.g. Malooly et al., 2013). Accuracy on repetition trials $(M=.94, S D=.04)$ was significantly higher than on switch trials $(M=.92, S D=.04), t(78)=4.11, p<.001$, $d=0.46$. RT on repetition trials $(M=1320, S D=196)$ was significantly lower than on switch trials $(M=$ $1460, S D=223), t(78)=14.79, p<.001, d=1.66$. This confirms the presence of overall accuracy and RT switch costs, and rules out a speed-accuracy tradeoff explaining differences on switch and repetition trials.

\section{Descriptive statistics}

Descriptive statistics and correlations for the switch costs and self-report measures are shown in Table 1. Additionally, Table 1 provides measures of spread for STAI-T and PSWQ scores. The lack of strong correlations between specific switch costs is consistent with previous studies (Genet et al., 2013; Malooly et al., 2013) and supports the decision to break down affective flexibility into different components. Additionally, no significant correlations were found 
Table 1. Descriptive statistics and bivariate correlations between study variables.

\begin{tabular}{|c|c|c|c|c|c|c|c|c|}
\hline Variable & 1 & 2 & 3 & 4 & 5 & 6 & 7 & 8 \\
\hline 1. N/NA Switch Cost (ms) & - & .08 & .14 & .17 & -.03 & -.14 & -.09 & $-.21^{\dagger}$ \\
\hline 2. N/A Switch Cost (ms) & & - & -.03 & $.21^{\dagger}$ & -.11 & -.07 & -.10 & -.09 \\
\hline 3. P/NA Switch Cost (ms) & & & - & .04 & .08 & .03 & .05 & .06 \\
\hline 4. P/A Switch Cost (ms) & & & & - & -.10 & $<.01$ & -.12 & -.07 \\
\hline 5. Baseline STAI-T & & & & & - & $.86^{* * *}$ & $.82^{* * *}$ & $.80^{* * *}$ \\
\hline 6. Final STAI-T & & & & & & - & $.70^{* * *}$ & $.82^{* * *}$ \\
\hline 7. Baseline PSWQ & & & & & & & - & $.86^{* * *}$ \\
\hline 8. Final PSWQ & & & & & & & & - \\
\hline M & 71.97 & 173.06 & 165.87 & 173.58 & 42.10 & 44.30 & 49.46 & 51.41 \\
\hline$S D$ & 219.39 & 205.59 & 182.56 & 178.07 & 11.00 & 11.19 & 14.38 & 13.72 \\
\hline range & & & & & $24-72$ & $22-75$ & $21-80$ & 20-79 \\
\hline IQR & & & & & 16 & 11 & 23 & 21 \\
\hline Q1 & & & & & 33 & 38 & 38 & 41 \\
\hline Q2 & & & & & 41 & 43 & 50 & 52 \\
\hline Q3 & & & & & 49 & 49 & 61 & 62 \\
\hline
\end{tabular}

Note: $\mathrm{N} / \mathrm{NA}=$ negative non-affective; $\mathrm{N} / \mathrm{A}=$ negative affective; $\mathrm{P} / \mathrm{NA}=$ positive non-affective; $\mathrm{P} / \mathrm{A}=$ positive affective. $\mathrm{IQR}=$ interquartile range. ${ }^{\dagger} p<.08 .{ }^{*} p<.05 .{ }^{* *} p<.01 .{ }^{* * *} p<.001$.

between switch costs and concurrent (baseline) levels of STAI-T trait anxiety and PSWQ worry.

\section{Affective flexibility ${ }^{3}$}

\section{STAI-T anxiety}

Results of the hierarchical regression analysis on STAI$\mathrm{T}$ trait anxiety are summarised in Table 2. Adding negative non-affective switch costs and positive affective switch costs, on top of baseline anxiety, resulted in a significant change in $R^{2}=0.03, F(2,75)=$ 4.36, $p=.016$. Adding negative affective switch costs and positive non-affective switch costs for exploratory purposes, did not result in a further significant increase in $R^{2}<0.01, F(2,73)=0.46, p=.633$. Specifically, more efficient shifting of attention from affective towards non-affective aspects of negative information predicted higher STAI-T anxiety after seven weeks, $\beta=-0.14, \quad t=-2.47, \quad p=.016$. Additionally, less efficient shifting of attention from non-affective towards affective aspects of positive information predicted higher STAI-T anxiety after seven weeks, $\beta=0.11, t=2.02, p=.047$.

\section{PSWQ worry}

The results of the hierarchical regression analysis on PSWQ worry are summarised in Table 2 . Adding negative non-affective switch costs and positive affective

Table 2. Hierarchical regression analysis on STAI-trait anxiety and PSWQ worry.

\begin{tabular}{|c|c|c|c|c|c|c|c|c|c|c|}
\hline Model & Predictor & $b$ & $95 \% \mathrm{Cl}$ & $\beta$ & $t$ & $p$ & $R^{2}$ & $R^{2}$ change & $F$ & $p$ \\
\hline \multicolumn{11}{|c|}{ STAI-T trait anxiety } \\
\hline Step 1 & Baseline STAI-T & 0.88 & $0.76,0.99$ & 0.86 & 14.91 & $<.001$ & 0.74 & 0.74 & 222.2 & $<.001$ \\
\hline \multirow[t]{3}{*}{ Step 2} & Baseline STAI-T & 0.88 & $0.77,1.00$ & 0.87 & 15.61 & $<.001$ & 0.76 & 0.03 & 4.36 & .016 \\
\hline & N/NA switch cost & -0.007 & $-0.013,-0.001$ & -0.14 & -2.47 & .016 & & & & \\
\hline & P/A switch cost & 0.007 & $0.0001,0.014$ & 0.11 & 2.02 & .047 & & & & \\
\hline \multirow[t]{5}{*}{ Step 3} & Baseline STAI-T & 0.89 & $0.78,1.01$ & 0.88 & 15.44 & $<.001$ & 0.76 & 0.003 & 0.46 & .633 \\
\hline & N/NA switch cost & -0.007 & $-0.02,-0.001$ & -0.13 & -2.32 & .023 & & & & \\
\hline & P/A switch cost & 0.008 & $0.001,0.015$ & 0.13 & 2.15 & .035 & & & & \\
\hline & N/A switch cost & -0.003 & $-0.009,0.004$ & -0.05 & -0.85 & .398 & & & & \\
\hline & P/NA switch cost & -0.002 & $-0.009,0.005$ & -0.03 & -0.49 & .626 & & & & \\
\hline \multicolumn{11}{|c|}{ PSWQ worry } \\
\hline Step 1 & Baseline PSWQ & 0.82 & $0.71,0.93$ & 0.86 & 14.55 & $<.001$ & 0.73 & 0.73 & 211.6 & $<.001$ \\
\hline \multirow[t]{3}{*}{ Step 2} & Baseline PSWQ & 0.81 & $0.70,0.92$ & 0.85 & 14.74 & $<.001$ & 0.76 & 0.02 & 3.32 & .042 \\
\hline & N/NA switch cost & -0.009 & $-0.016,-0.002$ & -0.15 & -2.49 & 0.015 & & & & \\
\hline & P/A switch cost & 0.005 & $-0.004,0.014$ & 0.06 & 1.04 & 0.30 & & & & \\
\hline \multirow[t]{5}{*}{ Step 3} & Baseline PSWQ & 0.81 & $0.70,0.92$ & 0.85 & 14.37 & $<.001$ & 0.76 & 0.002 & 0.28 & .757 \\
\hline & N/NA switch cost & -0.009 & $-0.017,-0.002$ & -0.15 & -2.55 & .013 & & & & \\
\hline & P/A switch cost & 0.004 & $-0.005,0.014$ & 0.06 & 0.96 & .341 & & & & \\
\hline & N/A switch cost & 0.001 & $-0.007,0.009$ & 0.01 & 0.18 & .862 & & & & \\
\hline & P/NA switch cost & 0.003 & $-0.006,0.012$ & 0.04 & 0.74 & .465 & & & & \\
\hline
\end{tabular}

Note: $N / N A=$ negative non-affective; $N / A=$ negative affective; $P / N A=$ positive non-affective; $P / A=$ positive affective. 
switch costs, on top of baseline PSWQ worry, resulted in a significant change in $R^{2}=0.02, F(2,75)=3.32$, $p=.042$. However, when correcting for multiple testing because we ran separate hierarchical regression analysis for anxiety and worry (i.e. a $=.025$ ), this would not be considered significant. Adding negative affective switch costs and positive non-affective switch costs for exploratory purposes, did not result in a further significant increase in $R^{2}<$ $0.01, F(2,73)=0.28, p=.757$. Specifically, more efficient shifting of attention from affective towards non-affective aspects of negative information predicted higher PSWQ worry after seven weeks, $\beta=-0.15, t=-2.49, p=.015$.

\section{Discussion}

Whilst deficits in cognitive flexibility have been associated with anxiety and worry in theoretical models (Eysenck et al., 2007; Hirsch \& Mathews, 2012), there is minimal empirical research investigating the relationship between cognitive flexibility in the context of affective stimuli (i.e. affective flexibility) and anxiety or worry.

As predicted, we found that less efficient shifting (i.e. reduced flexibility) from processing the nonaffective to affective aspects of positive stimuli was associated with higher levels of STAI-T anxiety over time. This effect was small, but the relationship cannot be explained by a general cognitive flexibility deficit as it remained when controlling for the other switch costs. This result supports previous findings from cross-sectional research associating poorer affective flexibility with higher trait anxiety (Mărcuş et al., 2016). This finding also supports a growing body of research associating aberrant processing of positive information with anxiety (Taylor et al., 2011). However, our study extends previous findings by using a prospective design to evaluate the impact of affective flexibility on STAI-T anxiety over time. By breaking down affective flexibility into different components our study revealed more nuanced relationships with anxiety: that specific difficulties shifting from processing the non-affective to affective aspects of positive stimuli seem to be relevant. These specific results also coincide with previous studies linking affective positive switch costs with emotion regulation. For example, greater flexibility when shifting towards the affective aspects of positive stimuli has been associated with increased reappraisal effectiveness (Malooly et al., 2013) and decreased physiological arousal during a frightening experience (Hildebrandt et al., 2016). These findings will now need to be replicated in larger and more diverse samples, to confirm that it is indeed not general impairments in affective flexibility that can predict anxiety or worry, but rather specific difficulties shifting to processing affective aspects of positive information.

The role of altered processing of negative information in anxiety and worry, as compared to a focus on positive information, has been more extensively studied. Contrary to expectations, we found that greater flexibility when shifting attention away from processing the affective aspects of negative stimuli was associated with greater increases in STAI-T anxiety and PSWQ worry over time. Although this effect was small, it is inconsistent with the idea that anxiety and worry are associated with a general impairment in affective flexibility (Eysenck et al., 2007; Hirsch \& Mathews, 2012; Mărcuş et al., 2016). Instead, our findings may be understood by considering the vigilance-avoidance model of anxiety (Mogg et al., 2004) and empirical research associating heightened anxiety with greater attentional avoidance of negative information (Cisler \& Koster, 2010). Additionally, recent findings (Lester et al., 2019) show that a larger increase in attentional bias towards threat (but not away from threat) in children predicted a greater reduction in anxiety over time. This greater attentional bias to threat was suggested to facilitate learning in everyday life that an apparently threatening situation does not always lead to an aversive outcome (Lester et al., 2019), thus reducing anxiety over time, whereas avoiding processing of feared stimuli could lead to the persistence of anxiety symptoms because it prevents processing of feared stimuli so that extinction learning does not alter threat associations (Borkovec, 1994). It may be interesting for future studies to examine whether relatively efficient shifting of attention from affective towards non-affective aspects of negative information relates to (behavioural and cognitive) avoidance observed in anxiety.

Another observation from our findings was that affective flexibility appeared to predict levels of STAI-T anxiety and PSWQ worry after a seven-week period, when taking into account baseline levels, but was not related to concurrent levels of anxiety and worry. This is consistent with previous findings in an adolescent sample (Han et al., 2016), where executive function deficits, as measured with a 
Wisconsin Card Sorting Test, predicted anxiety levels over a two-year period but did not relate to concurrent anxiety levels. It is possible that individual differences in cognitive control may affect how adaptively people deal with stressors, such that an impact on anxiety and worry becomes apparent over time in response to challenges. Over the course of the seven weeks of our study, levels of STAI-T anxiety and PSWQ worry increased in our predominantly student sample, possibly in response to the extended mild stress of the university year, allowing effects of affective flexibility to be observed.

Although increased cognitive flexibility, or efficient mental set-shifting, is generally thought to be adaptive, our findings corroborate previous results (Genet et al., 2013) that suggest that more efficient set-shifting is not adaptive per se. The idea that most cognitive processing styles should not be considered universally adaptive or maladaptive is one of the central premises of the cognitive model of psychological resilience (Parsons et al., 2016). This model of resilience focuses on the ability to dynamically apply appropriate cognitive processing relevant to situational demands, perceived needs and personal goals. Parsons and colleagues argue that flexibility in the cognitive system is not adaptive per se, but that transitioning between flexibility and rigidity in processing style is important in promoting adaptive cognitive processing. Although still theoretical in nature, the authors propose an overarching "mapping" system that allocates contextually appropriate information processing, integrating information about the situation and personal goals, but also previous experience in dealing/coping with a situation (Parsons et al., 2016).

It is important to note that effects of affective flexibility on STAI-T anxiety were small and affective flexibility may therefore be an unlikely target for interventions by itself. There are many (cognitive) factors that contribute to individual differences and changes in anxiety, or sustained negative affect more generally. Any one process may explain only a small amount of variance. Together and in interaction such (cognitive) processes, e.g. cognitive flexibility and biases in information processing, can influence individuals' anxiety or distress levels. Similarly, clinical anxiety and mood disorders are very heterogeneous, with many factors that can contribute to the development and maintenance of such mental disorders. This means we need to put current effects of affective flexibility in perspective, such that affective flexibility is one of many factors influencing anxiety (and worry) levels and in itself has only limited influence on predicting anxiety and worry over time.

Several limitations to the study should be noted. Firstly, our sample consisted largely of a healthy young adult student population, which limits the generalisability of the findings to other populations, including clinical populations. Nonetheless, our sample included people reporting low, moderate, and higher levels of STAI-T trait anxiety and PSWQ worry. Secondly, although the STAI-T is usually described as a measure of trait anxiety, previous results suggest that the scale rather reflects negative affect in general (Bados et al., 2010). Therefore, our results may not strictly apply to trait anxiety (and worry), but rather relate to negative affect more generally. Thirdly, the affective flexibility task does not allow us to disentangle the relative contributions of different processes (e.g. inhibition, shifting) that are involved in cognitive flexibility (Monsell, 2003). Whilst this was not central to our research question, it may be interesting for future studies to investigate whether specific processes are driving these findings, using tasks that attempt to separate out the inhibition and shifting components of cognitive flexibility. Another limitation of the current study is the relatively short time frame, which limits our ability to draw strong conclusions about the predictive value of affective flexibility over longer time periods. In the current study, we aimed to examine whether individual differences in affective flexibility at one point, would predict changes in anxiety and worry across a couple of months. This is an important first step and future studies are required to measure levels of anxiety and worry and affective flexibility across a longer time period and unpick how these dynamic relationships unfold. Additionally, in order to make causal inferences it would also be beneficial to experimentally manipulate affective flexibility and observe the effects on anxiety/worry. Given that our hypotheses and analytical approach were not pre-registered, it is important to note that our findings are exploratory in nature and in need of replication. Nevertheless, our study is the first to investigate whether individual differences in affective flexibility measured at one point, can predict the experience of anxiety/worry symptoms over time, and future studies can build from this.

In summary, reduced flexibility when shifting towards processing the affective aspects of positive 
stimuli was associated with greater increases in STAI-T anxiety over time. Whilst previous research has largely focused on maladaptive processing of negative stimuli, this finding suggests that inflexible processing of positive stimuli may also play a role in anxiety. Contrary to expectations, we found that more efficient shifting away from processing the affective aspects of negative stimuli was associated with greater increases in STAI-T anxiety and PSWQ worry. This finding could be viewed in light of empirical evidence and theoretical models that associate anxiety and worry with greater avoidance of negative information, especially at longer exposure to such information. The effects in the current study were small and will need to be replicated in larger, representative samples, but our findings are a first indication that anxiety may not be associated with general impairments in cognitive flexibility. This highlights that affective flexibility needs to be broken down into different components to be able to reveal and understand more nuanced relationships. This could have implications for theories, such as Attentional Control Theory, which predict that inflexibility, regardless of the context, is associated with anxiety. Our findings corroborate the idea that cognitive processing styles can be adaptive or maladaptive depending on situational demands, as laid out in the cognitive model of psychological resilience (Parsons et al., 2016).

\section{Notes}

1. An a priori power analysis for a hierarchical linear multiple regression, based on the $\mathrm{R}^{2}$ increase when testing four predictors and total number of predictors is five (i.e. including baseline anxiety/worry), assuming a medium effect size $f^{2}=0.15$ and power of 0.8 , shows the necessary sample size is 85 . When testing two predictors (i.e. switch costs of interest) and the total number of predictors is three, the necessary sample size is 68 .

2. We also administered the Connor-Davidson Resilience Scale and the Cognitive Emotion Regulation Questionnaire. The Hassles and Uplifts Scale was administered once a week in between the two test sessions. However, these will not be used to answer the current research questions.

3. Exploratory hierarchical regression analyses with accuracy-based switch costs showed that none of these switch costs significantly predicted anxiety or worry.

\section{Authorship}

All authors contributed to the study concept and design. Data collection, analysis and interpretation was performed by E. Twivy, under the supervision of M. Grol and E. Fox. E. Twivy and M. Grol drafted the paper, and E. Fox. provided critical reviews. All authors edited and approved the final version of the paper for submission.

\section{Open practices statement}

The data for this study are available on the Open Science Framework: https://osf.io/2vb8x/?view_only= 88f9ea5a2cb741af9d1fdf5a65d177c1

\section{Disclosure statement}

No potential conflict of interest was reported by the author(s).

\section{Funding}

This research was supported by a grant awarded to E. Fox from the FP7 Ideas: European Research Council under the European Union's Seventh Framework Programme (FP7/2007-2013) / ERC grant agreement no. [324176] for the CogBIAS project, and a studentship awarded to E. Twivy, funded by the Medical Research Council and Department of Experimental Psychology, University of Oxford. M. Grol is currently supported by a postdoctoral fellowship of the Research Foundation Flanders (FWO2017/PDO/201).

\section{References}

Ansari, T. L., Derakshan, N., \& Richards, A. (2008). Effects of anxiety on task switching: Evidence from the mixed antisaccade task. Cognitive, Affective, \& Behavioral Neuroscience, 8 (3), 229-238. https://doi.org/10.3758/CABN.8.3.229

Bados, A., Gómez-Benito, J., \& Balaguer, G. (2010). The state-trait anxiety inventory, trait version: Does it really measure anxiety? Journal of Personality Assessment, 92(6), 560-567. https://doi.org/10.1080/00223891.2010.513295

Berggren, N., \& Derakshan, N. (2013). Attentional control deficits in trait anxiety: Why you see them and why you don't. Biological Psychology, 92(3), 440-446. https://doi.org/10. 1016/j.biopsycho.2012.03.007

Borkovec, T. D. (1994). The nature, functions, and origins of worry. In G. Davey, \& F. Tallis (Eds.), Worrying: Perspectives on theory, assessment and treatment (pp. 5-34). Wiley.

Cisler, J. M., \& Koster, E. H. W. (2010). Mechanisms of attentional biases towards threat in anxiety disorders: An integrative review. Clinical Psychology Review, 30(2), 203-216. https:// doi.org/10.1016/j.cpr.2009.11.003

Clarke, P., MacLeod, C., \& Shirazee, N. (2008). Prepared for the worst: Readiness to acquire threat bias and susceptibility to elevate trait anxiety. Emotion, 8(1), 47-57. https://doi.org/ 10.1037/1528-3542.8.1.47

Demanet, J., Liefooghe, B., \& Verbruggen, F. (2011). Valence, arousal, and cognitive control: A voluntary task-switching study. Frontiers in Psychology, 2. https://doi.org/10.3389/ fpsyg.2011.00336 
Eysenck, M. W., Derakshan, N., Santos, R., \& Calvo, M. G. (2007). Anxiety and cognitive performance: Attentional control theory. Emotion, 7(2), 336-353. https://doi.org/10.1037/ 1528-3542.7.2.336

Genet, J. J., Malooly, A. M., \& Siemer, M. (2013). Flexibility is not always adaptive: Affective flexibility and inflexibility predict rumination use in everyday life. Cognition \& Emotion, 27(4), 685-695. https://doi.org/10.1080/02699931.2012.733351

Geurts, H. M., Corbett, B., \& Solomon, M. (2009). The paradox of cognitive flexibility in autism. Trends in Cognitive Sciences, 13 (2), 74-82. https://doi.org/10.1016/j.tics.2008.11.006

Han, G., Helm, J., lucha, C., Zahn-Waxler, C., Hastings, P. D., \& Klimes-Dougan, B. (2016). Are executive functioning deficits concurrently and predictively associated with depressive and anxiety symptoms in adolescents? Journal of Clinical Child \& Adolescent Psychology, 45(1), 44-58. https://doi.org/ 10.1080/15374416.2015.1041592

Hildebrandt, L. K., McCall, C., Engen, H. G., \& Singer, T. (2016). Cognitive flexibility, heart rate variability, and resilience predict fine-grained regulation of arousal during prolonged threat. Psychophysiology, 53(6), 880-890. https://doi.org/10. 1111/psyp.12632

Hirsch, C. R., \& Mathews, A. (2012). A cognitive model of pathological worry. Behaviour Research and Therapy, 50(10), 636646. https://doi.org/10.1016/j.brat.2012.06.007

Kashdan, T. B., \& Rottenberg, J. (2010). Psychological flexibility as a fundamental aspect of health. Clinical Psychology Review, 30(7), 865-878. https://doi.org/10.1016/j.cpr.2010.03.001

Lang, P. J., Bradley, M. M., \& Cuthbert, B. N. (1999). International affective picture system (IAPS): Technical manual and affective ratings (Vol. 2). The Center for Research in Psychophysiology, University of Florida.

Lee, J. K., \& Orsillo, S. M. (2014). Investigating cognitive flexibility as a potential mechanism of mindfulness in generalized anxiety disorder. Journal of Behavior Therapy and Experimental Psychiatry, 45(1), 208-216. https://doi.org/10. 1016/j.jbtep.2013.10.008

Lester, K. J., Lisk, S. C., Carr, E., Patrick, F., \& Eley, T. C. (2019). Associations between attentional bias and interpretation bias and change in school concerns and anxiety symptoms during the transition from primary to secondary school. Journal of Abnormal Child Psychology, 47, 1521-1532. https://doi.org/10.1007/s10802-019-00528-3

Malooly, A. M., Genet, J. J., \& Siemer, M. (2013). Individual differences in reappraisal effectiveness: The role of affective flexibility. Emotion, 13(2), 302-313. https://doi.org/10.1037/ a0029980

Mărcuş, O., Stanciu, O., MacLeod, C., Liebregts, H., \& Visu-Petra, L. (2016). A FISTful of emotion: Individual differences in trait anxiety and cognitive-affective flexibility during preadolescence. Journal of Abnormal Child Psychology, 44(7), 12311242. https://doi.org/10.1007/s10802-015-0110-z

Meyer, T. J., Miller, M. L., Metzger, R. L., \& Borkovec, T. D. (1990). Development and validation of the penn state worry questionnaire. Behaviour Research and Therapy, 28(6), 487-495. https://doi.org/10.1016/0005-7967(90)90135-6

Mogg, K., Bradley, B., Miles, F., \& Dixon, R. (2004). Brief report time course of attentional bias for threat scenes: Testing the vigilance-avoidance hypothesis. Cognition and Emotion, 18(5), 689-700. https://doi.org/10.1080/02699930341000158

Monsell, S. (2003). Task switching. Trends in Cognitive Sciences, 7 (3), 134-140. https://doi.org/10.1016/S1364-6613(03)00028-7

Parsons, S. (2020). Splithalf: robust estimates of split half reliability (Version 4). figshare. https://doi.org/10.6084/m9. figshare.11956746.v4

Parsons, S., Kruijt, A. W., \& Fox, E. (2016). A cognitive model of psychological resilience. Journal of Experimental Psychopathology, 7(3), 296-310. https://doi.org/10.5127/jep. 053415

Roberts, B. W., Luo, J., Briley, D. A., Chow, P. I., Su, R., \& Hill, P. L. (2017). A systematic review of personality trait change through intervention. Psychological Bulletin, 143(2), 117141. https://doi.org/10.1037/bul0000088

Spielberger, C. D., Gorsuch, R. L., Lushene, R., Vagg, P. R., \& Jacobs, G. A. (1983). State-trait anxiety inventory for adults. Mind Garden.

Taylor, C. T., Bomyea, J., \& Amir, N. (2011). Malleability of attentional bias for positive emotional information and anxiety vulnerability. Emotion, 11(1), 127-138. https://doi.org/10. 1037/a0021301 